\title{
Nonlinearly Balanced Boolean Functions and Their Propagation Characteristics (Extended Abstract)
}

\author{
Jennifer Seberry * Xian-Mo Zhang ${ }^{\star}$ and Yuliang Zheng * \\ Department of Computer Science \\ University of Wollongong, Wollongong, NSW 2522, Australia \\ \{jennie, xianmo, yoliang\}ecs.not.edu.an
}

\begin{abstract}
Three of the most important criteria for cryptographically strong Boolean functions are the balancedness, the nonlinearity and the propagation criterion. This paper studies systematic methods for constructing Boolean functions satisfying some or all of the three criteria. We show that concatenating, splitting, modifying and multiplying sequences can yield balanced Boolean functions with a very high nonlinearity. In particular, we show that balanced Boolean functions obtained by modifying and multiplying sequences achieve nonlinearity higher than that attainable by any previously known construction method. We also present methods for constructing highly nonlinear balanced Boolean functions satisfying the propagation criterion with respect to all but one or three vectors. A technique is developed to transform the vectors where the propagation criterion is not satisfied in such a way that the functions constructed satisfy the propagation criterion of high degree while preserving the balancedness and nonlinearity of the functions. The algebraic degrees of functions constructed are also discussed, together with example illustrating the various constructions.
\end{abstract}

\section{Preliminaries}

Let $f$ be a function on $V_{n}$. The $(1,-1)$-sequence defined by $\left((-1)^{f\left(\alpha_{0}\right)},(-1)^{f\left(\alpha_{1}\right)}\right.$, $\left.\ldots,(-1)^{f\left(\alpha_{2 n}-1\right)}\right)$ is called the sequence of $f$, and the $(0,1)$-sequence defined by $\left(f\left(\alpha_{0}\right), f\left(\alpha_{1}\right), \ldots, f\left(\alpha_{2^{n}-1}\right)\right)$ is called the trith table of $f$, where $\alpha_{i}, 0 \leq i \leq$ $2^{n}-1$, denotes the vector in $V_{n}$ whose integer representation is $i$. A $(0,1)-$ sequence $((1,-1)$-sequence) is said balanced if it contains an equal number of zeros and ones (ones and minus ones). A function is balanced if its sequence is balanced.

- Supported in part by the Australian Research Council under the reference numbers A49130102, A9030136, A49131885 and A49232172.

** Supported in part by the Australian Research Council under the reference number A49130102.

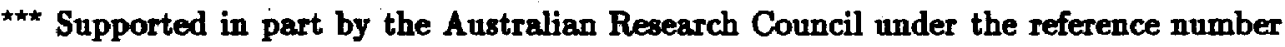
A49232172. 
The Hamming weight of a $(0,1)$-sequence (or vector) $\alpha$, denoted by $W(\alpha)$, is the number of ones in $\alpha$. The Hamming distance between two sequences $\alpha$ and $\beta$ of the same length, denoted by $d(\alpha, \beta)$, is the number of positions where the two sequences differ. Given two functions $f$ and $g$ on $V_{n}$, the Hamming distance between them is defined as $d(f, g)=d\left(\xi_{f}, \xi_{g}\right)$, where $\xi_{f}$ and $\xi_{g}$ are the truth tables of $f$ and $g$ respectively. The nonlinearity of $f$, denoted by $N_{f}$, is the minimal Hamming distance between $f$ and all affine functions on $V_{n}$, i.e., $N_{f}=\min _{i=0,1, \ldots, 2^{n+1}-1} d\left(f, \varphi_{i}\right)$ where $\varphi_{0}, \varphi_{1}, \ldots, \varphi_{2^{n+1}-1}$ denote the affine functions on $V_{n}$.

A $(1,-1)$-matrix $H$ of order $n$ is called a Hadamard matrix if $H H^{t}=n I_{n}$, where $H^{t}$ is the transpose of $H$ and $I_{n}$ is the identity matrix of order $n$. It is well known that the order of a Hadamard matrix is 1,2 or divisible by 4 [11]. A special kind of Hadamard matrix, called Sylvester-Hadamard matrix or WalshHadamard matrix, will be relevant to this paper. A Sylvester-Hadamard matrix of order $2^{n}$, denoted by $H_{n}$, is generated by the following recursive relation

$$
H_{0}=1, H_{n}=\left[\begin{array}{rr}
1 & 1 \\
1 & -1
\end{array}\right] \otimes H_{n-1}, n=1,2, \ldots
$$

where $\otimes$ denotes the Kronecker product. Note that $H_{n}$ can be represented as $H_{n}=H_{s} \otimes H_{t}$ for any $s$ and $t$ with $s+t=n$.

Sylvester-Hadamard matrices are closely related to linear functions, as is shown in the following lemma.'

Lemma 1. Write $H_{n}=\left[\begin{array}{c}\ell_{0}^{\prime} \\ \ell_{1} \\ \vdots \\ \ell_{2^{n}-1}\end{array}\right]$ where $\ell_{i}$ is a row of $H_{n}$. Then $\ell_{i}$ is the sequence of $h_{i}=\left\langle\alpha_{i}, x\right\rangle$, a linear function, where $\alpha_{i}$ is a vector in $V_{n}$ whose integer representation is $i$ and $x=\left(x_{1}, \ldots, x_{n}\right)$. Conversely the sequence of any linear function on $V_{n}$ is a row of $H_{n}$.

From Lemma 1 the rows of $H_{n}$ comprise the sequences of all linear functions on $V_{n}$. Consequently the rows of $\pm H_{n}$ comprise the sequences of all affine functions on $V_{n}$.

The following notation is very useful in obtaining the functional representation of a concatenated sequence. Let $\delta=\left(i_{1}, i_{2}, \ldots, i_{p}\right)$ be a vector in $V_{p}$. Then $D_{\delta}$ is a function on $V_{p}$ defined by

$$
D_{6}\left(y_{1}, y_{2}, \ldots, y_{p}\right)=\left(y_{1} \oplus i_{1} \oplus 1\right) \cdots\left(y_{p} \oplus i_{p} \oplus 1\right) .
$$

We now introduce the concept of bent functions.

Definition 2. A function $f$ on $V_{n}$ is called a bent function if

$$
2^{-\frac{n}{2}} \sum_{x \in V_{n}}(-1)^{f(x) \oplus\langle\beta, x\rangle}= \pm 1
$$

for all $\beta \in V_{n}$. Here $f(x) \oplus\langle\beta, x\rangle$ is regarded as a real-valued function. The sequence of a bent function is called a bent sequence. 
From the definition we can see that bent functions on $V_{n}$ exist only when $n$ is even. It was Rothaus who first introduced and studied bent functions in $1960 \mathrm{~s}$, although his pioneering work was not published in the open literature until some ten years later [10]. Applications of bent functions to digital communications, coding theory and cryptography can be found in such as $[2,4,7]$.

The following result can be found in an excellent survey of bent functions by Dillon [5].

Lemma 3. Let $f$ be a function on $V_{n}$, and let $\xi$ be the sequence of $f$. Then the following four statements are equivalent:

(i) $f$ is bent.

(ii) $\langle\xi, \ell\rangle= \pm 2^{\frac{1}{2} n}$ for any affine sequence $\ell$ of length $2^{n}$.

(iii) $f(x) \oplus f(x \oplus \alpha)$ is balanced for any non-zero vector $\alpha \in V_{n}$.

(iv) $f(x) \oplus\langle\alpha, x\rangle$ assumes the value one $2^{n-1} \pm 2^{\frac{1}{2} n-1}$ times for any $\alpha \in V_{n}$.

By (iv) of Lemma 3, if $f$ is a bent function on $V_{n}$, then $f(x) \oplus h(x)$ is also a bent function for any affine function $h$ on $V_{n}$. This property will be employed in constructing highly nonlinear balanced functions to be described in Section 4 .

In this paper we are concerned with the propagation criterion whose formal definition follows (see also $[1,9]$ ).

Definition 4. Let $f$ be a function on $V_{n}$. We say that $f$ satisfies

1. the propagation criterion with respect to a non-zero vector $\alpha$ in $V_{n}$ if $f(x) \oplus$ $f(x \oplus \alpha)$ is a balanced function.

2. the propagation criterion of degree $k$ if it satisfies the propagation criterion with respect to all $\alpha \in V_{n}$ with $1 \leq W(\alpha) \leq k$.

Note that the SAC is equivalent to the propagation criterion of degree 1. Also note that the perfect nonlinearity studied by Meier and Staffelbach [6] is equivalent to the propagation criterion of degree $n$.

\section{Properties of Balancedness and Nonlinearity}

This section presents a number of results related to balancedness and nonlinearity. These include upper bounds for nonlinearity and properties of concatenated and split sequences. Due to the limit on space, proofs for some of the results are left to the full version of the paper [13]. tion.

The following lemma is very useful in calculating the nonlinearity of a func-

Lemma 5. Let $f$ and $g$ be functions on $V_{n}$ whose sequences are $\xi_{f}$ and $\xi_{g}$ respectively. Then the distance between $f$ and $g$ can be calculated by $d(f, g)=$ $2^{n-1}-\frac{1}{2}\left\langle\xi_{f}, \xi_{g}\right\rangle$. 
Corollary 6. A function on $V_{n}$ attains the upper bound for nonlinearities, $2^{n-1}-$ $2^{\frac{1}{2} n-1}$, if and only if it is bent.

From Corollary 6, balanced functions can not attain the upper bound for nonlinearities, namely $2^{n-1}-2^{\frac{1}{2} n-1}$. A slightly improved upper bound for the nonlinearities of balanced functions can be obtained by noting the fact that a balanced function assumes the value one an even number of times.

Corollary 7. Let $f$ be a balanced function on $V_{n}(n \geq 3)$. Then the nonlinearity $N_{f}$ of $f$ is given by

$$
N_{f} \leq\left\{\begin{array}{l}
2^{n-1}-2^{\frac{1}{2} n-1}-2, n \text { even } \\
\left\lfloor\left\lfloor 2^{n-1}-2^{\frac{1}{2} n-1}\right\rfloor\right\rfloor, n \text { odd }
\end{array}\right.
$$

where $\lfloor\lfloor x\rfloor\rfloor$ denotes the maximum even integer less than or equal to $x$.

The following lemma, first proved in [12], gives the lower bound of the nonlinearity of a function obtained by concatenating the sequences of two functions.

Lemma 8. Let $f_{1}$ and $f_{2}$ be functions on $V_{n}$, and let $g$ be a function on $V_{n+1}$ defined by

$$
g\left(u, x_{1}, \ldots, x_{n}\right)=(1 \oplus u) f_{1}\left(x_{1}, \ldots, x_{n}\right) \oplus u f_{2}\left(x_{1}, \ldots, x_{n}\right) .
$$

Suppose that $\xi_{1}$ and $\xi_{2}$, the sequences of $f_{1}$ and $f_{2}$ respectively, satisfy $\left\langle\xi_{1}, \ell\right\rangle \leq P_{1}$ and $\left\langle\xi_{2}, \ell\right\rangle \leq P_{2}$ for any affine sequence $\ell$ of length $2^{n}$, where $P_{1}$ and $P_{2}$ are positive integers. Then the nonlinearity of $g$ satisfies $N_{g} \geq 2^{n}-\frac{1}{2}\left(P_{1}+P_{2}\right)$.

As bent functions do not exist on $V_{2 k+1}$, an interesting question is what functions on $V_{2 k+1}$ are highly nonlinear. The following result, as a special case of Lemma 8, shows that such functions can be obtained by concatenating bent sequences. This construction has also been discovered by Meier and Staffelbach in [6].

Corollary 9. In the construction (1), if both $f_{1}$ and $f_{2}$ are bent functions on $V_{2 k}$, then $N_{g} \geq 2^{2 k}-2^{k}$.

A similar result can be obtained when sequences of four functions are concatenated.

Lemma 10. Let $f_{0}, f_{1}, f_{2}$ and $f_{3}$ be functions on $V_{n}$ whose sequences are $\xi_{0}$, $\xi_{1}, \xi_{2}$ and $\xi_{3}$ respectively. Assume that $\left\langle\xi_{i}, \ell\right\rangle \leq P_{i}$ for each $0 \leq i \leq 3$ and for each affine sequence $\ell$ of length $2^{n}$, where each $P_{i}$ is a positive integer. Let $g$ be a function on $V_{n+2}$ defined by

$$
g(y, x)=\bigoplus_{i=0}^{3} D_{\alpha_{i}}(y) f_{i}(x)
$$

where $y=\left(y_{1}, y_{2}\right), x=\left(x_{1}, \ldots, x_{n}\right)$ and $\alpha_{i}$ is a vector in $V_{2}$ whose integer representation is $i$. Then $N_{g} \geq 2^{n+1}-\frac{1}{2}\left(P_{0}+P_{1}+P_{2}+P_{3}\right)$. In particular, when $n$ is even and $f_{0}, f_{1}, f_{2}$ and $f_{3}$ are all bent functions on $V_{n}, N_{g} \geq 2^{n+1}-2^{\frac{1}{2} n+1}$. 
We have discussed the concatenation of sequences of functions including bent functions. The following lemma deals with the other direction, namely splitting bent sequences.

Lemma 11. Let $f\left(x_{1}, x_{2}, \ldots, x_{2 k}\right)$ be a bent function on $V_{2 k}, \eta_{0}$ be the sequence of $f\left(0, x_{2}, \ldots, x_{2 k}\right)$, and $\eta_{1}$ be the sequence of $f\left(1, x_{2}, \ldots, x_{2 k}\right)$. Then for any affine sequence $\ell$ of length $2^{2 k-1}$, we have $-2^{k} \leq\left\langle\eta_{0}, \ell\right\rangle \leq 2^{k}$ and $-2^{k} \leq\left\langle\eta_{1}, \ell\right\rangle \leq$ $2^{k}$.

A consequence of Lemma 11 is that the nonlinearity of $f\left(0, x_{2}, \ldots, x_{2 k}\right)$ and $f\left(1, x_{2}, \ldots, x_{2 k}\right)$ is at least $2^{2 k-2}-2^{k-1}$. It is interesting to note that concatenating and splitting bent sequences both achieve the same nonlinearity.

Splitting bent sequences can also result in balanced functions. Let $\ell_{i}$ be the $i$ th row of $H_{k}$ where $i=0,1, \ldots, 2^{k}-1$. Note that $\ell_{0}$ is an all-one sequence while $\ell_{1}, \ell_{2}, \ldots, \ell_{2^{k}-1}$ are all balanced sequences. The concatenation of the rows, $\left(\ell_{0}, \ell_{1}, \ldots, \ell_{2^{k}-1}\right)$, is a bent sequence [1]. Denote by $f\left(x_{1}, x_{2}, \ldots, x_{2 k}\right)$ the function corresponding to the bent sequence. Let $\xi$ be the second half of the bent sequence, namely, $\xi=\left(\ell_{2^{k-1}}, \ell_{2^{k-1}+1}, \ldots, \ell_{2^{k}-1}\right)$. Then $\xi$ is the sequence of $f\left(1, x_{2}, \ldots, x_{2 k}\right)$. Since all $\ell_{i}, i=2^{k-1}, 2^{k-1}+1, \ldots, 2^{k}-1$, are balanced, $f\left(1, x_{2}, \ldots, x_{2 k}\right)$ is a balanced function. The nonlinearity of the function is at least $2^{2 k-2}-2^{k-1}$.

By permuting $\left\{\ell_{2^{k-1}}, \ell_{2^{k-1}+1}, \ldots, \ell_{2^{k}-1}\right\}$, we obtain a new balanced sequence $\xi^{\prime}=\left(\ell_{2^{k-1}}^{\prime}, \ell_{2^{k-1}+1}^{\prime}, \ldots, \ell_{2^{k}-1}^{\prime}\right)$ that has the same nonlinearity as that of $\xi$. Now let $\xi^{\prime \prime}=\left(e_{2^{k-1}} \ell_{2^{k-1}}^{\prime}, e_{2^{k-1}+1} \ell_{2^{k-1}+1}^{\prime}, \ldots, e_{2^{k}-1} \ell_{2^{k}-1}^{\prime}\right)$, where each $e_{i}$ is independently selected from $\{1,-1\} . \xi^{\prime \prime}$ is also a balanced sequence with the same nonlinearity. The total number of balanced sequences obtained by permuting and changing signs is $2^{2^{k-1}} \cdot 2^{k-1}$ !. These sequences are all different from one another but have the same nonlinearity.

\section{Highly Nonlinear Balanced Functions}

Note that a bent sequence on $V_{2 k}$ contains $2^{2 k-1}+2^{k-1}$ ones and $2^{2 k-1}-2^{k-1}$ zeros, or vice versa. As is observed by Meier and Staffelbach [6], changing $2^{k-1}$ positions in a bent sequence yields a balanced function having a nonlinearity of at least $2^{2 k-1}-2^{k}$. This nonlinearity is the same as that obtained by concatenating four bent sequences of length $2^{2 k-2}$ (see Lemma 10).

It is well-known that the maximum nonlinearity of functions on $V_{n}$ coincides with the covering radius of the first order binary Reed-Muller code $R(1, n)$ of length $2^{n}$, many results on covering radius of $R(1, n)$ (see [3]) have direct implications on the nonlinearity of functions. In particular, using a result of [8], we can construct unbalanced functions on $V_{2 k+1}, k \geq 7$, whose nonlinearity is at least $2^{2 k}-\frac{108}{128} 2^{k}$, a higher value than $2^{2 k}-2^{k}$ achieved by the construction in Corollary 9. One might tempt to think that modifying the sequences in [8] would result in balanced functions with a higher nonlinearity than that obtained by concatenating or splitting bent sequences. We find that it is not the case. We 
take $V_{15}$ for an example. The Hamming weight of the sequences on $V_{15}$, which have the largest nonlinearity of 16276 , is 16492 . Changing 54 positions makes them balanced. The nonlinearity of the resulting functions is 16222 , smaller than 16256 achieved by concatenating two bent sequences of length $2^{14}$ (see Corollary 9).

In the following we show how to modify bent sequences of length $2^{2 k}$ constructed from Hadamard matrices in such a way that the resulting functions are balanced and have a much higher nonlinearity than that attainable by concatenating four bent sequences. This result, in conjunction with sequences in [8], allows us to construct balanced functions on $V_{2 k+15}, k \geq 7$, that have a higher nonlinearity than that achieved by concatenating or splitting bent sequences.

\subsection{On $V_{z k}$}

Note that an even number $n \geq 4$ can be expressed as $n=4 t$ or $n=4 t+2$, where $t \geq 1$. As the first step towards our goal, we have the following lemma whose proof is left to the full paper [13]:

\section{Lemma 12. For any integer $t \geq 1$ there exists}

(i) a balanced function $f$ on $V_{4 t}$ such that $N_{f} \geq 2^{4 t-1}-2^{2 t-1}-2^{t}$, (ii) a balanced function $f$ on $V_{4 t+2}$ such that $N_{g} \geq 2^{4 t+1}-2^{2 t}-2^{t}$.

With the above result as a basis, we consider an iterative procedure to further improve the nonlinearity of a function constructed. Note that an even number $n \geq 4$ can be expressed as $n=2^{m}, m \geq 2$, or $n=2^{s}(2 t+1), s \geq 1$ and $t \geq 1$.

Consider the case when $n=2^{m}, m \geq 2$. We start with the bent sequence obtained by concatenating the rows of $\bar{H}_{2^{m-1}}$. The sequence consists of $2^{2^{m-1}}$ sequences of length $2^{2^{m-1}}$. Now we replace the all-one leading sequence with a bent sequence of the same length, which is obtained by concatenating the rows of $H_{2 m-2}$. The length of the new leading sequence becomes $2^{2^{m-2}}$. It is replaced by another bent sequence of the same length. This replacing process is continued until the length of the all-one leading sequence is $2^{2}=4$. To finish the procedure, we replace the leading sequence $(1,1,1,1)$ with $(1,-1,1,-1)$. The last replacement makes the entire sequence balanced. By induction on $s=2,3,4, \ldots$, it can be proved that the nonlinearity of the function obtained is at least

$$
2^{2^{m}-1}-\frac{1}{2}\left(2^{2^{m-1}}+2^{2^{m-2}}+\cdots+2^{2^{2}}+2 \cdot 2^{2}\right) .
$$

The modifying procedure for the case of $n=2^{s}(2 t+1), s \geq 1$ and $t \geq 1$, is the same as that for the case of $n=2^{m}, m \geq 2$, except for the last replacement. In this case, the replacing process is continued until the length of the all-one leading sequence is $2^{2 t+1}$. The last leading sequence is replaced by $\ell_{0}^{*}=$ $\left(e_{2^{t}}, e_{2^{t}+1}, \ldots, e_{2^{t+1}-1}\right)$, the second half of the bent sequence $\left(e_{0}, e_{1}, \ldots, e_{2^{t+1}-1}\right)$, where each $\epsilon_{i}$ is a row of $H_{t+1}$. Again by induction on $s=1,2,3, \ldots$, it can be proved that the nonlinearity of the resulting function is at least

$$
2^{2^{\circ}(2 t+1)-1}-\frac{1}{2}\left(2^{2^{s-1}(2 t+1)}+2^{2^{s-2}(2 t+1)}+\cdots+2^{2(2 t+1)}+2^{2 t+1}+2^{t+1}\right) .
$$


We have completed the proof for the following

Theorem 13. For any even number $n \geq 4$, there exists a balanced function $f^{*}$ on $V_{n}$ whose nonlinearity is

$$
N_{f^{*}} \geq\left\{\begin{array}{c}
2^{2^{m}-1}-\frac{1}{2}\left(2^{2^{m-1}}+2^{2^{m-2}}+\cdots+\right. \\
\left.2^{2^{2}}+2 \cdot 2^{2}\right), \\
2^{2^{*}(2 t+1)-1}-\frac{1}{2}\left(2^{2^{2-1}(2 t+1)}+2^{2^{*-3}(2 t+1)}+\cdots+\right. \\
\left.2^{2(2 t+1)}+2^{2 t+1}+2^{t+1}\right), \quad n=2^{m},
\end{array}\right.
$$

Let $\zeta=\left(\zeta_{0}, \zeta_{1}, \ldots, \zeta_{2^{k}-1}\right)$ be a sequence of length $2^{2 k}$ obtained by modifying a bent sequence. Permuting and changing signs discussed in Section 2 can also be applied to $\zeta$. In this way we obtain in total $2^{2^{k}} \cdot 2^{k}$ ! different balanced functions, all of which have the same nonlinearity. Even more functions can be obtained by observing the fact that the leading sequence $\zeta_{0}$ has exactly the same structure as the large sequence $\zeta$, and hence permuting and changing signs can also be applied to $\zeta_{0}$.

\subsection{On $V_{2 k+1}$}

Lemma 14. Let $f_{1}$ be a function on $V_{s}$ and $f_{2}$ be a function on $V_{t}$. Then $f_{1}\left(x_{1}, \ldots, x_{s}\right) \oplus f_{2}\left(y_{1}, \ldots, y_{t}\right)$ is a balanced function on $V_{s+t}$ if either $f_{1}$ or $f_{2}$ is balanced.

Let $\xi_{1}$ be the sequence of $f_{1}$ on $V_{s}$ and $\xi_{2}$ be the sequence of $f_{2}$ on $V_{t}$. Then it is easy to verify that the Kronecker product $\xi_{1} \otimes \xi_{2}$ is the sequence of $f_{1}\left(x_{1}, \ldots, x_{s}\right) \oplus f_{2}\left(y_{1}, \ldots, y_{t}\right)$.

Lemma 15. Let $f_{1}$ be a function on $V_{s}$ and $f_{2}$ be a function on $V_{t}$. Let $g$ be a function on $V_{s+t}$ defined by

$$
g\left(x_{1}, \ldots, x_{s}, y_{1}, \ldots, y_{s}\right)=f_{1}\left(x_{1}, \ldots, x_{s}\right) \oplus f_{2}\left(y_{1}, \ldots, y_{t}\right) .
$$

Suppose that $\xi_{1}$ and $\xi_{2}$, the sequences of $f_{1}$ and $f_{2}$ respectively, satisfy $\left\langle\xi_{1}, \ell\right\rangle \leq P_{1}$ and $\left\langle\xi_{2}, \ell\right\rangle \leq P_{2}$ for any affine sequence $\ell$ of length $2^{n}$, where $P_{1}$ and $P_{2}$ are positive integers. Then the nonlinearity of $g$ satisfies $N_{g} \geq 2^{s+t-1}-\frac{1}{2} P_{1} \cdot P_{2}$.

Let $\xi_{1}$ be a balanced sequence of length $2^{2 k}$ that is constructed using the method in the proof of Theorem 13, where $k \geq 2$, Let $\xi_{2}$ be a sequence of length $2^{15}$ obtained by the method of [8]. Note that the nonlinearity of $\xi_{2}$ is 16276 , and there are 13021 such sequences. Denote by $f_{1}$ the function corresponding to $\xi_{1}$ and by $f_{2}$ the function corresponding to $\xi_{2}$. Let

$$
f\left(x_{1}, \ldots, x_{2 k}, x_{2 k+1}, \ldots, x_{2 k+15}\right)=f_{1}\left(x_{1}, \ldots, x_{2 k}\right) \oplus f_{2}\left(x_{2 k+1}, \ldots, x_{2 k+15}\right)(3)
$$


Theorem 16. The function $f$ defined by (3) is a balanced function on $V_{2 k+15}$, $k \geq 2$, whose nonlinearity is at least

$$
N_{f} \geq\left\{\begin{array}{c}
2^{2^{m}+14}-108\left(2^{2^{m-1}}+2^{2^{m-2}}+\cdots+\right. \\
\left.2^{2^{2}}+2 \cdot 2^{2}\right), \\
2^{2^{s}(2 t+1)+14}-108\left(2^{2^{o-1}(2 t+1)}+2^{2^{s-2}(2 t+1)}+\cdots+2 k=2^{m},\right. \\
\left.2^{2(2 t+1)}+2^{2 t+1}+2^{t+1}\right), \quad 2 k=2^{s}(2 t+1) .
\end{array}\right.
$$

Proof. Let $\xi=\xi_{1} \otimes \xi_{2}$. Then $\xi$ is the sequence of $f$. Let $\ell$ be an arbitrary affine sequence of length $2^{2 k+15}$. Then $\ell= \pm \ell_{1} \otimes \ell_{2}$, where $\ell_{1}$ is a linear sequence of length $2^{2 k}$ and $\ell_{2}$ is a linear sequence of length $2^{15}$. Thus

$\left\langle\xi_{1}, \ell_{1}\right\rangle \leq \begin{cases}2^{2^{m-1}}+2^{2^{m-2}}+\cdots+2^{2^{2}}+2 \cdot 2^{2}, & 2 k=2^{m}, \\ 2^{2^{s-1}(2 t+1)}+2^{2^{s-2}(2 t+1)}+\cdots+2^{2(2 t+1)}+2^{2 t+1}+2^{t+1}, & 2 k=2^{s}(2 t+1) .\end{cases}$

and

$$
\left\langle\xi_{2}, \ell_{2}\right\rangle \leq 2 \cdot\left(2^{14}-16276\right)=216
$$

By Lemma 15, the theorem is true.

The nonlinearity of a function on $V_{2 k+15}$ constructed in this section is larger than that obtained by concatenating or splitting bent sequences for all $k \geq 7$.

\section{Constructing Highly Nonlinear balanced Functions Satisfying High Degree Propagation Criterion}

This section presents two methods for constructing highly nonlinear balanced functions satisfying the propagation criterion.

\subsection{Basic Construction}

On $V_{2 k+1}$ Let $f$ be a bent function on $V_{2 k}$, and let $g$ be a function on $V_{2 k+1}$ defined by

$$
\begin{aligned}
& g\left(x_{1}, x_{2}, \ldots, x_{2 k+1}\right) \\
& =\left(1 \oplus x_{1}\right) f\left(x_{2}, \ldots, x_{2 k+1}\right) \oplus x_{1}\left(1 \oplus f\left(x_{2}, \ldots, x_{2 k+1}\right)\right) \\
& =x_{1} \oplus f\left(x_{2}, \ldots, x_{2 k+1}\right) .
\end{aligned}
$$

Lemma 17. The function $g$ defined in (4) satisfies the propagation criterion with respect to all non-zero vectors $\gamma \in V_{2 k+1}$ with $\gamma \neq(1,0, \ldots, 0)$.

Proof. Let $\gamma=\left(a_{1}, a_{2}, \ldots, a_{2 k+1}\right) \neq(1,0, \ldots, 0)$ and let $x=\left(x_{1}, x_{2}, \ldots, x_{2 k+1}\right)$. Then $g(x) \oplus g(x \oplus \gamma)=a_{1} \oplus f\left(x_{2}, \ldots, x_{2 k+1}\right) \oplus f\left(x_{2} \oplus a_{2}, \ldots, x_{2 k+1} \oplus a_{2 k+1}\right)$. Since $f$ is a bent function, $f\left(x_{2}, \ldots, x_{2 k+1}\right) \oplus f\left(x_{2} \oplus a_{2}, \ldots, x_{2 k+1} \oplus a_{2 k+1}\right)$ is balanced for all $\left(a_{2}, \ldots, a_{2 k+1}\right) \neq(0, \ldots, 0)$ (see (iii) of Lemma 3). Thus $g(x) \oplus g(x \oplus \gamma)$ is balanced for all $\gamma=\left(a_{1}, a_{2}, \ldots, a_{2 k+1}\right) \neq(1,0, \ldots, 0)$. 
From Corollary 9, the nonlinearity of the function $g$ defined by (4) satisfies $N_{g} \geq 2^{2 k}-2^{k}$. Furthermore, by Lemma 14, $g$ is balanced. Thus we have

Corollary 18. The function $g$ defined by (4) is balanced and satisfies the propagation criterion with respect to all non-zero vectors $\gamma \in V_{2 k+1}$ with $\gamma \neq(1,0, \ldots, 0)$. The nonlinearity of $g$ satisfies $N_{g} \geq 2^{2 k}-2^{k}$.

On $V_{2 k}$ Let $f$ be a bent function on $V_{2 k-2}$ and let $g$ be a function on $V_{2 k}$ obtained from $f$ in the following way:

$$
\begin{aligned}
& g\left(x_{1}, x_{2}, x_{3}, \ldots, x_{2 k}\right) \\
& =\left(1 \oplus x_{1}\right)\left(1 \oplus x_{2}\right) f\left(x_{3}, \ldots, x_{2 k}\right) \oplus\left(1 \oplus x_{1}\right) x_{2}\left(1 \oplus f\left(x_{3}, \ldots, x_{2 k}\right)\right) \\
& \quad x_{1}\left(1 \oplus x_{2}\right)\left(1 \oplus f\left(x_{3}, \ldots, x_{2 k}\right)\right) \oplus x_{1} x_{2} f\left(x_{3}, \ldots, x_{2 k}\right) \\
& =x_{1} \oplus x_{2} \oplus f\left(x_{3}, \ldots, x_{2 k}\right) .
\end{aligned}
$$

Lemma 19. The function $g$ defined in (5) satisfies the propagation criterion with respect to all but three non-zero vectors in $V_{2 k}$. The three vectors where the propagation criterion is not satisfied are $\gamma_{1}=(1,0,0, \ldots, 0), \gamma_{2}=(0,1,0, \ldots, 0)$, and $\gamma_{3}=\gamma_{1} \oplus \gamma_{2}=(1,1,0, \ldots, 0)$.

Proof. Let $\gamma=\left(a_{1}, a_{2}, \ldots, a_{2 k}\right)$ be a non-zero vector in $V_{2 k}$ differing from $\gamma_{1}, \gamma_{2}$ and $\gamma_{3}$. Also let $x=\left(x_{1}, \ldots, x_{2 k}\right)$. Then we have $g(x) \oplus g(x \oplus \gamma)=a_{1} \oplus a_{2} \oplus$ $f\left(x_{3}, \ldots, x_{2 k}\right) \oplus f\left(x_{3} \oplus a_{3}, \ldots, x_{2 k} \oplus a_{2 k}\right)$. Since $f$ is a bent function on $V_{2 k-2}$ and $\left(a_{3}, \ldots, a_{2 k}\right) \neq(0, \ldots, 0), f\left(x_{3}, \ldots, x_{2 k}\right) \oplus f\left(x_{3} \oplus a_{3}, \ldots, x_{2 k} \oplus a_{2 k}\right)$ is balanced, from which it follows that $g(x) \oplus g(x \oplus \gamma)$ is balanced for any non-zero vector $\gamma$ in $V_{2 k}$ differing from $\gamma_{1}, \gamma_{2}$ and $\gamma_{3}$. This proves the lemma.

Since $x_{1} \oplus x_{2}$ is balanced on $V_{2}, g$ is balanced on $V_{2 k}$. On the other hand, by Lemma 8, we have $N_{g} \geq 2^{2 k-1}-2^{k}$. Thus we have the following result:

Corollary 20. The function $g$ defined by (5) is balanced and satisfies the propagation criterion with respect to all non-zero vectors $\gamma \in V_{2 k}$ with $\gamma \neq\left(c_{1}, c_{2}, 0, \ldots, 0\right)$, where $c_{1}, c_{2} \in G F(2)$. The nonlinearity of $g$ satisfies $N_{g} \geq 2^{2 k-1}-2^{k}$.

\subsection{Moving Vectors Around}

Though functions constructed according to (4) or (5) satisfy the propagation criterion with respect to all but one or three non-zero vectors, they are not interesting in practical applications. We show that through linear transformation of input coordinates, the vectors where the propagation criterion is not satisfied can be transformed while the balancedness and nonlinearity of the functions are preserved. In particular, the vectors can be transformed into vectors having a high Hamming weight. In this way we obtain highly nonlinear balanced functions satisfying the high degree propagation criterion.

Let $f$ be a function on $V_{n}, A$ a nondegenerate matrix of order $n$ with entries from $G F(2)$, and $b$ a vector in $V_{n}$. Then $f^{*}(x)=f(x A \oplus b)$ defines a new function on $V_{n}$, where $x=\left(x_{1}, x_{2}, \ldots, x_{n}\right)$. It can be proved that the algebraic degree and the nonlinearity of $f^{*}$ is the same as those of $f$. In addition, $f^{*}$ is balanced iff $f$ is balanced. 


\section{On $V_{2 k+1}$}

Theorem 21. For any non-zero vector $\gamma^{*} \in V_{2 k+1}(k \geq 1)$, there exist balanced functions on $V_{2 k+1}$ satisfying the propagation criterion with respect to all nonzero vectors $\gamma \in V_{2 k+1}$ with $\gamma \neq \gamma^{*}$. The nonlinearities of the functions are at least $2^{2 k}-2^{k}$.

Proof. Let $f$ be a bent function and let $g$ be the function constructed by (4). From linear algebra we know that for any bases $B_{1}$ and $B_{2}$ of the vector space $V_{2 k+1}$, where $B_{1}=\left\{\alpha_{j} \mid j=1, \ldots, 2 k+1\right\}$ and $B_{2}=\left\{\beta_{j} \mid j=1, \ldots, 2 k+1\right\}$, there exists a unique nondegenerate matrix $A$ of order $2 k+1$ with entries from $G F(2)$ such that $\alpha_{j} A=\beta_{j}, j=1, \ldots, 2 k+1$. In particular, this is true when $\alpha_{1}=\gamma^{*}$ and $\beta_{1}=(1,0, \ldots, 0)$. Let $x=\left(x_{1}, x_{2}, \ldots, x_{n}\right)$ and let $g^{*}$ be the function obtained from $g$ by employing linear transformation on the input coordinates of $g$ :

$$
g^{*}(x)=g(x A) .
$$

Since $A$ is nondegenerate, $g^{*}$ is balanced and has the same nonlinearity as that of $g$. Now we show that $g^{*}$ satisfies the propagation criterion with respect to all non-zero vectors except $\gamma^{*}$.

Let $\gamma$ be a non-zero vector in $V_{2 k+1}$ with $\gamma \neq \gamma^{*}$. Consider the following function $g^{*}(x) \oplus g^{*}(x \oplus \gamma)=g(x A) \oplus g(x A \oplus \gamma A)=g(y) \oplus g(y \oplus \gamma A)$ where $y=x A$. Note that $A$ is nondegenerate and thus $y$ runs through $V_{2 k+1}$ while $x$ runs through $V_{2 k+1}$. Since $\gamma \neq \gamma^{*}$ we have $\gamma A \neq(1,0, \ldots, 0)$. From (iii) of Lemma $3, g(y) \oplus g(y \oplus \gamma A)$ is balanced and hence $g^{*}(x) \oplus g^{*}(x \oplus \gamma)$ is balanced. Consequently, $g^{*}$ satisfies the propagation criterion with respect to all non-zero vectors in $V_{2 k+1}$ but $\gamma^{*}$. This completes the proof.

As a consequence of Theorem 21 , we obtain, by letting $\gamma^{*}=(1,1, \ldots, 1)$, highly nonlinear balanced functions on $V_{2 k+1}$ satisfying the propagation criterion of degree $2 k$. This is described in the following:

Corollary 22. Let $f$ be $a$ bent function on $V_{2 k}$ and let $g^{*}\left(x_{1}, \ldots, x_{2 k+1}\right)=x_{1} \oplus$ $f\left(x_{1} \oplus x_{2}, x_{1} \oplus x_{3}, \ldots, x_{1} \oplus x_{2 k+1}\right)$. Then $g^{*}$ is a balanced function on $V_{2 k+1}$ and satisfies the propagation criterion of degree $2 k$. The nonlinearity of $g^{*}$ satisfies $N_{g^{*}} \geq 2^{2 k}-2^{k}$.

\section{On $V_{z k}$}

Theorem 23. For any non-zero vectors $\gamma_{1}^{*}, \gamma_{2}^{*} \in V_{2 k}(k \geq 2)$ with $\gamma_{1}^{*} \neq \gamma_{2}^{*}$, there exist balanced functions on $V_{2 k}$ satisfying the propagation criterion with respect to all but three non-zero vectors in $V_{2 k}$. The three vectors where the propagation criterion is not satisfied are $\gamma_{1}^{*}, \gamma_{2}^{*}$ and $\gamma_{1}^{*} \oplus \gamma_{2}^{*}$. The nonlinearities of the functions are at least $2^{2 k-1}-2^{k}$.

Proof. The proof is essentially the same as that for Theorem 21. The major difference lies in the selection of bases $B_{1}=\left\{\alpha_{j} \mid j=1, \ldots, 2 k\right\}$ and $B_{2}=\left\{\beta_{j} \mid j=\right.$ 
$1, \ldots, 2 k\}$. By linear algebra, we can let $\alpha_{1}=\gamma_{1}^{*}, \alpha_{2}=\dot{\gamma}_{2}^{*}, \beta_{1}=(1,0,0, \ldots, 0)$, and $\beta_{2}=(0,1,0, \ldots, 0)$. By the same reasoning as in the proof of Theorem 21, we can see that $g^{*}$ defined by $g^{*}(x)=g(x A)$ satisfies the propagation criterion with respect to all but the following three non-zero vectors in $V_{2 k}: \gamma_{1}^{*}, \gamma_{2}^{*}$ and $\gamma_{1}^{*} \oplus \gamma_{2}^{*}$. Here $x=\left(x_{1}, x_{2}, \ldots, x_{2 k}\right), g(x)=x_{1} \oplus x_{2} \oplus f\left(x_{3}, \ldots, x_{2 k}\right)$, and $f$, a bent function on $V_{2 k-2}$, are all the same as in (5), and $A$ is the unique nondegenerate matrix such that $\alpha_{j} A=\beta_{j}, j=1, \ldots, 2 k$.

Similarly to the case on $V_{2 k+1}$, we can obtain highly nonlinear balanced functions satisfying the high degree propagation criterion, by properly selecting vectors $\gamma_{1}^{*}$ and $\gamma_{2}^{*}$. Unlike the case on $V_{2 k+1}$, however, the degree of propagation criterion the functions can achieve is $\frac{4}{3} k$, but not $2 k-1$. The construction method is described in the following corollary.

Corollary 24. Suppose that $2 k=3 t+c$ where $c=0,1$ or 2 . Then there exist balanced functions on $V_{2 k}$ that satisfy the propagation criterion of degree $2 t-1$ (when $c=0$ or 1 ), or $2 t$ (when $c=2$ ). The nonlinearities of the functions are at least $2^{2 k-1}-2^{k}$.

Proof. Set $c_{1}=0, c_{2}=1$ if $c=1$ and set $c_{1}=c_{2}=\frac{1}{2} c$ otherwise. Let $\gamma_{1}^{*}=$ $\left(a_{1}, \ldots, a_{3 t+c}\right)$ and $\gamma_{2}^{*}=\left(b_{1}, \ldots, b_{3 t+c}\right)$, where

$$
\begin{gathered}
a_{j}= \begin{cases}1 & \text { for } j=1, \ldots, 2 t+c_{1}, \\
0 & \text { for } j=2 t+c_{1}+1, \ldots, 3 t+c .\end{cases} \\
b_{j}= \begin{cases}0 \text { for } j=1, \ldots, t+c_{1} \\
1 \text { for } j=t+c_{1}+1, \ldots, 3 t+c .\end{cases}
\end{gathered}
$$

By Theorem 23 there exists a balanced function $g^{*}$ on $V_{2 k}$ satisfying the propagation criterion with respect to all but three non-zero vectors in $V_{2 k}$. The three vectors are $\gamma_{1}^{*}, \gamma_{2}^{*}$ and $\gamma_{1}^{*} \oplus \gamma_{2}^{*}$. The nonlinearity of $g^{*}$ satisfies $N_{g^{*}} \geq$ $2^{2 k-1}-2^{k}$.

Note that $W\left(\gamma_{1}^{*}\right)=2 t+c_{1}, W\left(\gamma_{2}^{*}\right)=2 t+c_{2}$, and $W\left(\gamma_{1}^{*} \oplus \gamma_{2}^{*}\right)=2 t+2 c_{1}=$ $2 t+c$. The minimum among the three weights is $2 t+c_{1}$. Therefore, for any nonzero vector $\gamma \in V_{2 k}$ with $W(\gamma) \leq 2 t+c_{1}-1$, we have $\gamma \neq \gamma_{1}^{*}, \gamma_{2}^{*}$ or $\gamma_{1}^{*} \oplus \gamma_{2}^{*}$. By Theorem 23, $g^{*}(x) \oplus g^{*}(x \oplus \gamma)$ is balanced. From this we conclude that $g^{*}$ satisfies the propagation criterion of order $2 t+c_{1}-1$. The proof is completed by noting that $c_{1}=0$ if $c=0$ or 1 and $c_{1}=1$ if $c=2$.

In the full paper [13] we shall show that functions obtained by (4) and (5) can achieve a wide range of algebraic degrees, namely $2, \ldots, k$ and $2, \ldots$, $k-1$ respectively. We shall also provide two concrete examples to illustrate our construction methods. 


\section{References}

1. ADAMS, C. M., AND TAVARES, S. E. Generating and counting binary bent sequences. IEEE Transactions on Information Theory IT-36 No. 5 (1990), 11701173.

2. ADAms, C. M., AND TAVARES, S. E. The use of bent sequences to achieve higherorder strict avalanche criterion. Technical Report, TR 90-013, Department of Electrical Engineering, Queen's University, 1990.

3. Cohen, G. D., Karpovsky, M. G., H. F. Mattson, J., and Schatz, J. R. Covering radius - survey and recent results. IEEE Transactions on Information Theory IT-31, 3 (1985), 328-343.

4. Detombe, J., AND TAVARES, S. Constructing large cryptographically strong S-boxes. In Advances in Cryptology - AUSCRYPT'92 (1993), Springer-Verlag, Berlin, Heidelberg, New York. to appear.

5. Dillon, J. F. A survey of bent functions. The NSA Technical Journal (1972), 191-215. (unclassified).

6. Meier, W., AND STAFFElBach, O. Nonlinearity criteria for cryptographic functions. In Advances in Cryptology - EUROCRYPT'89 (1990), vol. 434, Lecture Notes in Computer Science, Springer-Verlag, Berlin, Heidelberg, New York, pp. 549-562.

7. Nyberg, K. Perfect nonlinear S-boxes. In Advances in Cryptology - EUROCRYPT'91 (1991), vol. 547, Lecture Notes in Computer Science, Springer-Verlag, Berlin, Heidelberg, New York, pp. 378-386.

8. Patterson, N. J., ANd WiedemanN, D. H. The covering radius of the $\left(2^{15}, 16\right)$ Reed-Muller code is at least 16276. IEEE Transactions on Information Theory IT-29, 3 (1983), 354-356.

9. Preneel, B., Leekwick, W. V., Linden, L. V., Govaerts, R., and VandeWALLE, J. Propagation characteristics of boolean functions. In Advances in Cryptology - EUROCRYPT'9O (1991), vol. 437, Lecture Notes in Computer Science, Springer-Verlag, Berlin, Heidelberg, New York, pp. 155-165.

10. Rothaus, O. S. On "bent" functions. Journal of Combinatorial Theory Ser. A, 20 (1976), 300-305.

11. SEBERRY, J., AND YAMADA, M. Hadamard matrices, sequences, and block designs. In Contemporary Design Theory: A Collection of Surveys, J. H. Dinitz and D. R. Stinson, Eds. John Wiley \& Sons, Inc, 1992, ch. 11, pp. 431-559.

12. SEBERRY, J., AND ZHANG, X. M. Highly nonlinear 0-1 balanced functions satisfying strict avalanche criterion. In Advances in Cryptology - AUSCRYPT'92 (1993), Springer-Verlag, Berlin, Heidelberg, New York. to appear.

13. SeberRY, J., ZhANG, X. M., AND ZHENG, Y. Nonlinearity and propagation characteristics of halanced boolean functions. Submitted for Publication, 1993. 